\title{
Volvariella (Pluteacae, Basidiomycota) en el Norte de la Argentina
}

\author{
Volvariella (Pluteaceae, Basidiomycota) from Northen Argentina
}

\author{
Nicolás Niveiro ${ }^{1,2,4}$, Natalia Andrea Ramirez ${ }^{1,2}$, Orlando Fabián Popoff ${ }^{1,2}$ \& Edgardo Omar Albertó ${ }^{3}$
}

\begin{abstract}
Resumen
Se describen e ilustran tres especies de Volvariella para el Norte de la Argentina: $V$. taylorii, conocida para las selvas de Yungas y Paranaense; V. subxerophytica, conocida exclusivamente para los bosques xerófilos de la región chaqueña y $V$. pusilla encontrada en las provincias del Chaco y Tucumán. Las dos primeras se caracterizan por poseer volva castaña grisácea y superficie del píleo fibrillosa mientras que la última se caracteriza por su volva blanca grisácea y superficie del píleo glabra a muy delicadamente fibrillosa. Se registra por primera vez para Argentina a $V$. taylorii y se amplía el área de distribución de $V$. subxerophytica para el sector oriental de la región chaqueña. Es importante destacar que $V$. subxerophytica era conocida sólo por el material tipo y mediante los nuevos ejemplares hallados fue posible mejorar la descripción original.

Palabras clave: Agaricomycetes, Fungi, taxonomía, V. subxerophytica, V. taylorii.
\end{abstract}

\begin{abstract}
Three species of Volvariella from Northern Argentina are illustrated and described. Volvariella taylorii, known from the Yungas and the Paranaense Rain Forests; $V$. subxerophytica, known exclusively from the xerophytic forest from "chaqueña" region, and $V$. pusilla which was found in Chaco and Tucumán provinces. The two former species are characterized by having brownish-grayish volva and the fibrillose pileus, and the later by its whitish-grayish volva and the finely and delicately fibrillose surface of the pileus. Volvariella taylorii is recorded for the first time from Argentina and the area of distribution of $V$. subxerophytica is expanded towards the oriental area of the "chaqueña" region. It is important to mention that $V$. subxerophytica was only known by the type material and that in this work the original description is improved with data obtained from new specimens.
\end{abstract}

Key words: Agaricomycetes, Fungi, taxonomy, V. subxerophytica, V. taylorii.

\section{Introducción}

El género Volvariella Speg. (Pluteaceae Agaricales) se caracteriza por presentar basidiomas vaginatoides, es decir que presenta una volva típicamente sacciforme en la base del pie y ausencia de anillo (Largent \& Baroni 1988). Además tiene laminillas libres, blanquecinas al principio, coloreándose de rosado en la madurez con una trama himenoforal convergente, caracteres que la ubican dentro de la familia Pluteaceae (Shaffer 1957; Singer 1986). Recientemente Justo et al. (2011) analizaron las relaciones filogenéticas de la familia Pluteaceae, comprobando que Volvariella en el sentido tradicional sensu Singer (1986) conforma un grupo polifiletico. Según Singer (1986) Volvariella está distribuida en cinco grupos morfológicos bien definidos en base al tamaño y coloración de los basidiomas, el tamaño de las esporas y los caracteres de la pileipellis. De estos, el grupo conformado por V.gloiocephala (DC.) Boekhout \& Enderle y especies relacionadas se separó del resto de las especies de Volvariella, conformando el género Volvopluteus Vizzini, Contu \& Justo (Justo

\footnotetext{
${ }^{1}$ Instituto de Botánica del Nordeste, IBONE (UNNE-CONICET), Sargento Cabral 2131, CC 209, Corrientes Capital, CP 3400, Argentina.

${ }^{2}$ Universidad Nacional del Nordeste, Depto. Biología, Facultad de Ciencias Exactas y Naturales y Agrimensura, Av. Libertad 5470, Corrientes Capital, CP 3400, Argentina.

${ }^{3}$ Instituto de Investigaciones Biotecnológicas - Instituto Tecnológico de Chascomús, IIB-INTECH (UNSAM-CONICET), Cam. Circ. Laguna Km 6, Chascomús, Buenos Aires, CP 7130, Argentina.

${ }^{4}$ Autor para la correspondencia: niconiveiro@gmail.com
} 
et al. 2011). Por otro lado, las restantes especies de Volvariella conforman un clado monofilético, el cual no está relacionado con Pluteus Fr. y Volvopluteus, por lo que la familia Pluteaceae incluyendo Volvariella, seria polifilética. Asimismo, las relaciones entre las especies de Volvariella aún no están bien definidas respecto a las especies de otros géneros. En los análisis realizado por Moncalvo et al. (2002) se las relaciona con Schizophyllum Fr. y Fistulina Bull., mientras que Justo et al. (2011) lo hace con Camarophyllus (Fr.) P. Kumm. y Cantharocybe H.E. Bigelow \& A.H. Sm., relaciones que no están bien soportadas estadísticamente. Asimismo, estos géneros no presentan relación morfológica con Volvariella, por lo que la posición de este último dentro de los Agaricales aún no está esclarecida.

Actualmente, Volvariella comprende 50 especies de distribución mundial (Kirk et al. 2008; Justo et al. 2011), agrupadas principalmente en dos clados, uno conformado por $V$. volvacea (Bull.) Singer y $V$. bombycina (Schaeff.) Singer, y el otro por $V$. taylorii (Berk. \& Broome) Singer, V. pusilla (Pers.) Singer y especies relacionadas (Justo et al. 2011). Estas últimas se caracterizan por presentar basidiomas pequeños ( $<50 \mathrm{~mm}$ diám.), con hábito saprófito como descomponedores de hojarasca, diferenciándose entre ellas por caracteres como la coloración de la superficie del píleo y la volva (Singer 1986; Justo et al. 2011); sin embargo, ocasionalmente presentan dificultades para su identificación debido al solapamiento de los caracteres que las diferencian (Boekhout 1986).

En el norte argentino este género fue estudiado por Singer \& Digilio (1952), Singer $(1953,1989)$ y Raithelhuber (2004), conociéndose un total de cinco especies para la región (Niveiro \& Albertó 2012). De estas, Volvariella pusilla, V. taylorii y $V$. subxerophytica Singer presentan controversias acerca de su delimitación y han sido confundidas entre sí. El objetivo del presente trabajo es describir, ilustrar y analizar las diferencias entre estas tres especies en base a colecciones del norte argentino.

\section{Materiales y Métodos}

Se analizaron los materiales identificados como Volvariella depositados en los herbarios LIL y CTES, además de colecciones recientemente realizadas por los autores. Los especímenes fueron descritos macroscópicamente utilizando la terminología propuesta por Largent (1986). Para los colores se utilizó la terminología propuesta por Kornerup \& Wanscher (1978). Para las observaciones microscópicas se realizaron cortes a mano alzada montados en Hidróxido de Potasio (5\%), teñidos con Floxina (1\%), y reactivo de Melzer (Wright \& Albertó 2002). En el caso particular de las esporas se utilizan las siguientes abreviaturas: $\mathrm{x}=$ media aritmética del largo y ancho de las esporas, $\mathrm{Q}=$ rango de variación en el coeficiente entre la longitud y el ancho de las esporas, $\mathrm{Qx}=$ media de los valores de $\mathrm{Q}, \mathrm{n}=$ número de esporas medidas de $\mathrm{N}=$ número de basidiomas. Los autores de los nombres científicos se indican de acuerdo al Index Fungorum-Authors of Fungal Names (2016), las siglas de los herbarios según Thiers (permanentemente actualizado).

\section{Resultados y Discusión}

\section{Clave de las especies de Volvariella y Volvopluteus del norte argentino}

1. Superficie del píleo víscida, glabra, esporas mayores a $11 \mu \mathrm{m}$ de long.......

Volvopluteus cnemidophora

1'. Superficie del pileo seca, generalmente fibrillosa, esporas menores a $11 \mu \mathrm{m}$ de long. ........................2

2. Píleo mayor a $40 \mathrm{~mm}$ diám. ..................................................................... Volvariella bombycina

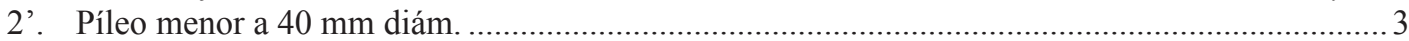

3. Volva blanca a gris clara. Esporas globosas a anchamente elipsoidales

1. Volvariella pusilla

3'. Volva negra a gris oscura o marrón. Esporas elipsoidales ................................................... 4

4. Superficie del píleo con el disco grisáceo oscuro, haciéndose rimoso hacia el margen pero llegando solo hasta la mitad del radio del píleo, margen gris claro a cremeo. Esporas $6,5-8,5 \times 5-6,5 \mu \mathrm{m}$. Pleurocistidios y queilocistidios ausentes o inconspicuos

2. Volvariella subxerophytica

4'. Superficie del píleo completamente rimosa hasta el margen, con fibrillas castaño grisáceas a negras. Esporas 5-7 × 3-4,8 $\mu \mathrm{m}$. Pleurocistidios y queilocistidios conspicuos 


\section{Taxonomía}

1. Volvariella pusilla (Pers.) Singer, Lilloa 22: 401, 1951 [1949].

Amanita pusilla Pers. Observationes mycologicae 2: 36,1800 [1799].

Volvariella parvula (Weinm.) Speg. Boletín de la Academia Nacional de Ciencias en Córdoba 28: 309, 1926.

Volvariella argentina Speg. Anales del Museo Nacional de Historia Natural Buenos Aires 6: 119, 1898.

Figs. 1; 2

Píleo hasta $20 \mathrm{~mm}$ de diám., convexo, volviéndose plano-convexo, levemente umbonado, a veces con el centro deprimido alrededor del umbo, glabro a muy delicadamente fibrilloso, blanco a grisáceo claro (1A1-1B1), con el centro levemente más oscuro (2B1), seco; margen entero, incurvado, estriado. Contexto delgado, blanco a gris claro (1A1-1B1). Olor y sabor no testeados. Laminillas apretadas a próximas, blancas (1A1), tornándose rosadas (7A2-8A2), margen liso, concoloro con los lados de las laminillas, libres, ventricosas, hasta $3 \mathrm{~mm}$ de ancho, con lamélulas. Estípite central, hasta $25 \times 3 \mathrm{~mm}$, cilíndrico, igual, blanco (1A1), glabro. Volva sacciforme, 2 o 4-lobada, hasta $7 \times 4 \mathrm{~mm}$, blanco sucio a gris claro (1B1-3B1), glabra o delicadamente pilosa. Tomento basal ausente. Anillo ausente. Esporada rosada pálida.

Esporas 4,5-6,5(-7) $\times 3,5-5,5 \mu \mathrm{m} ; \mathrm{x}=$ $5,9 \times 4,8 ; \mathrm{Q}=1,03-1,5 ; \mathrm{Q}_{\mathrm{x}}=1,22 ; \mathrm{n}=60 ;$ $\mathrm{N}=2$; globosas a anchamente elipsoidales, ovoides, hialinas a amarillas pálidas, con paredes finamente engrosadas, lisas, inamiliodes. Basidios 18-22 × 4-7 $\mu \mathrm{m}$; tetraesporados, claviformes, de paredes delgadas. Pleurocistidios 27-45 $\times$ 7-16 $\mu \mathrm{m}$, ventricosos, lageniformes a utriformes, hialinos, de paredes delgadas, poco abundantes. Queilocistidios 25-35 × 6-13 $\mu \mathrm{m}$, similares a los pleurocistidios, dispersos en el margen de la laminilla. Trama himenoforal convergente, hifas 3-7 $\mu \mathrm{m}$ diám. Pileipellis en un cutis de hifas no gelatinosas, postradas, entremezcladas, más o menos paralelas, 3-11 $\mu \mathrm{m}$ de diám., con
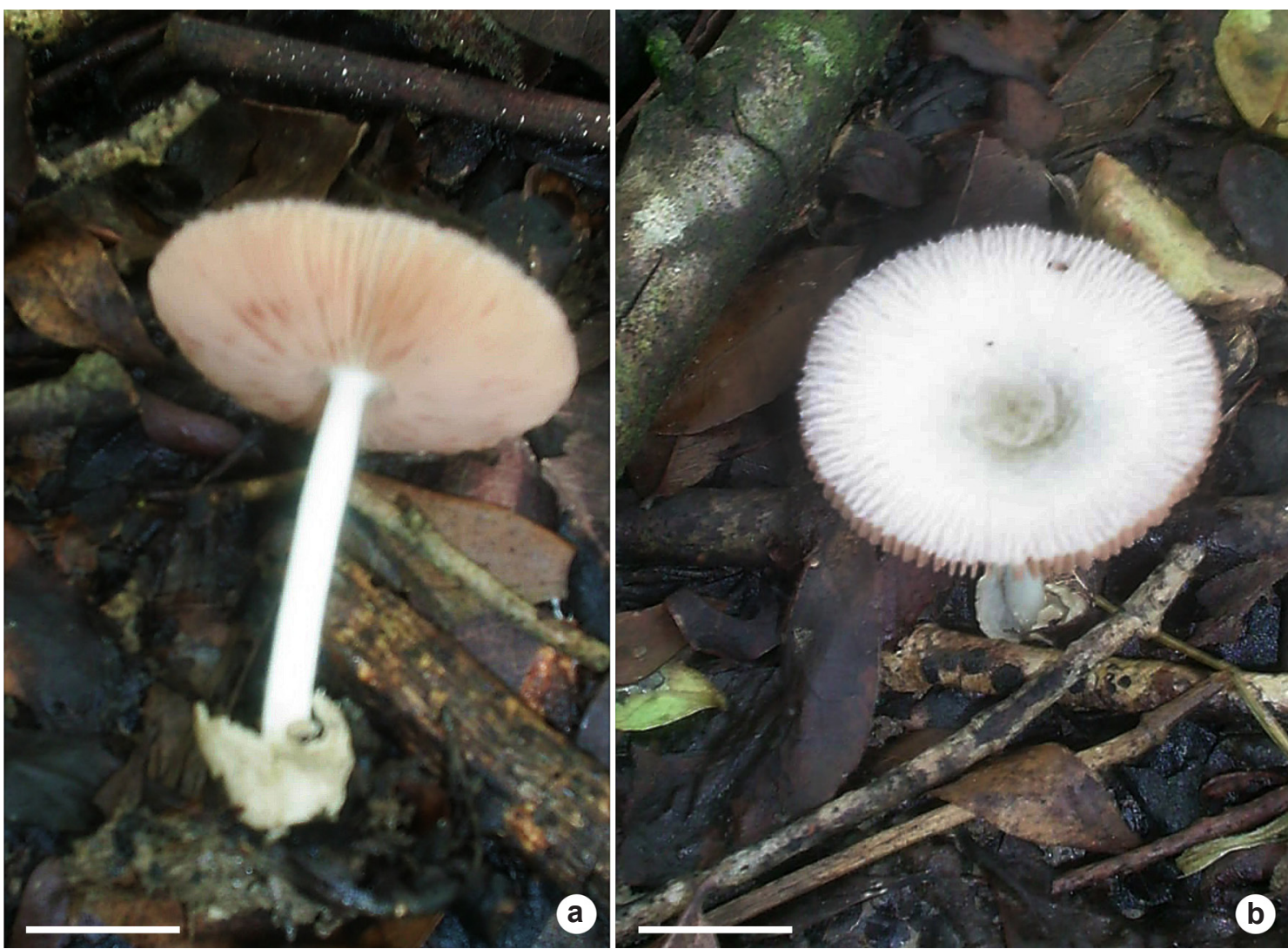

Figura 1 - Volvariella pusilla - a. vista de las laminillas, estípite y volva; b. vista de la superficie del píleo. Escala = $1 \mathrm{~cm}$. Figure 1 - Volvariella pusilla - a. view of lamellae, stipe and volva; b. view of pileus surface. $S c a l e=1 \mathrm{~cm}$. 
pigmentos castaños claros incrustados. Fíbulas ausentes.

Hábitat: Solitario, creciendo en el suelo entre la hojarasca.

Distribución: Ampliamente distribuida en regiones tropicales y templadas (Boekhout 1990). Conocida para África (Pegler 1977), Europa (Lange 1936, Kuhner \& Romagnesi 1953, Orton 1974), Norteamérica (Shaffer 1957), el Caribe (Pegler 1983) y Sudamérica (Spegazzini 1899; Singer \& Digilio 1952).

Material examinado: ARGENTINA. CHACO: San Fernando. Parque Municipal Caraguatá, IV.2008. Niveiro 309 (CTES). TUCUMÁN: San Miguel de Tucumán, Instituto Lillo, Ad terram nudam in horto. 12.IX.1949, Singer T119 (LIL).

Observaciones: Volvariella pusilla se caracteriza por su píleo de hasta $30 \mathrm{~mm}$ de diám., blanquecino a grisáceo claro y el estípite con volva blanca a gris clara que permanece de ese color en la madurez (Pegler 1983). Es una especie con una distribución muy amplia, en regiones templadas y tropicales de todo el mundo, incluso Shaffer (1962) considera a Volvariella argentina Speg., especie tipo del género descrita en la provincia de Buenos Aires (Spegazzini 1899, 1926; Singer 1950), como un sinónimo de esta especie.

Volvariella taylorii y V. psammophila Singer (Singer 1989) son dos especies similares, incluso la primera fue propuesta como una variedad dentro de otra especie: V. pusilla var. taylorii (Berk.) Boekhout (Boekhout 1986, 1990). Sin embargo, ambas especies presentan basidiomas más grandes, con coloraciones grisáceas en la superficie del píleo y en la volva (Singer 1989), caracteres que son considerados de importancia taxonómica para la delimitación de las especies de este género y los que la diferencian claramente de $V$. pusilla. Volvariella islagarciae Raithelh. es otra especie que podría confundirse con $V$. pusilla por su coloración clara, color de arena o algo más pálido y la volva blanquecina en estados juveniles (Raithelhuber 2000, 2004); sin embargo se diferencian claramente debido a que $V$. islagarciae tiene un porte mayor, de 40-50 mm diám., la superficie del píleo radialmente rasgada, la volva que se torna marrón en la madurez y las esporas de mayor tamaño, 7-9 $\times 5-5,5 \mu \mathrm{m}$ (Raithelhuber 2000, 2004). Volvariella hipopithys (Fr.) Shaffer es muy similar en el tamaño y coloración del basidioma, pero se diferencia en que presenta el estípite piloso, con pelos de hasta $450 \mu \mathrm{m}$ long. (Shaffer 1957; Boekhout 1986, 1990).

Volvariella platensis (Speg.) Shaffer, es otra especie descrita para Argentina en la misma región donde Spegazzini también coleccionó a $V$. argentina. Volvariella platensis se caracteriza por su píleo mediano de $25 \mathrm{~mm}$ de diám. de color grisáceo a cremeo, con fibrillas adpresas en la superficie del píleo; volva irregularmente lobulada, blanca (Spegazzini 1899) y esporas subglobosas a ovoides 5,7-7,5 × 4,4-5,9 $\mu \mathrm{m}$ (Shaffer 1962). Considerando las características mencionadas en la descripción original (Spegazzini 1899) y en el análisis realizado por Shaffer (1962) del material tipo, es muy probable que esta especie sea un sinónimo de $V$. pusilla debido a que no se observan diferencias morfológicas entre ambas especies. Sin embargo, para corroborar esto es necesario un análisis del
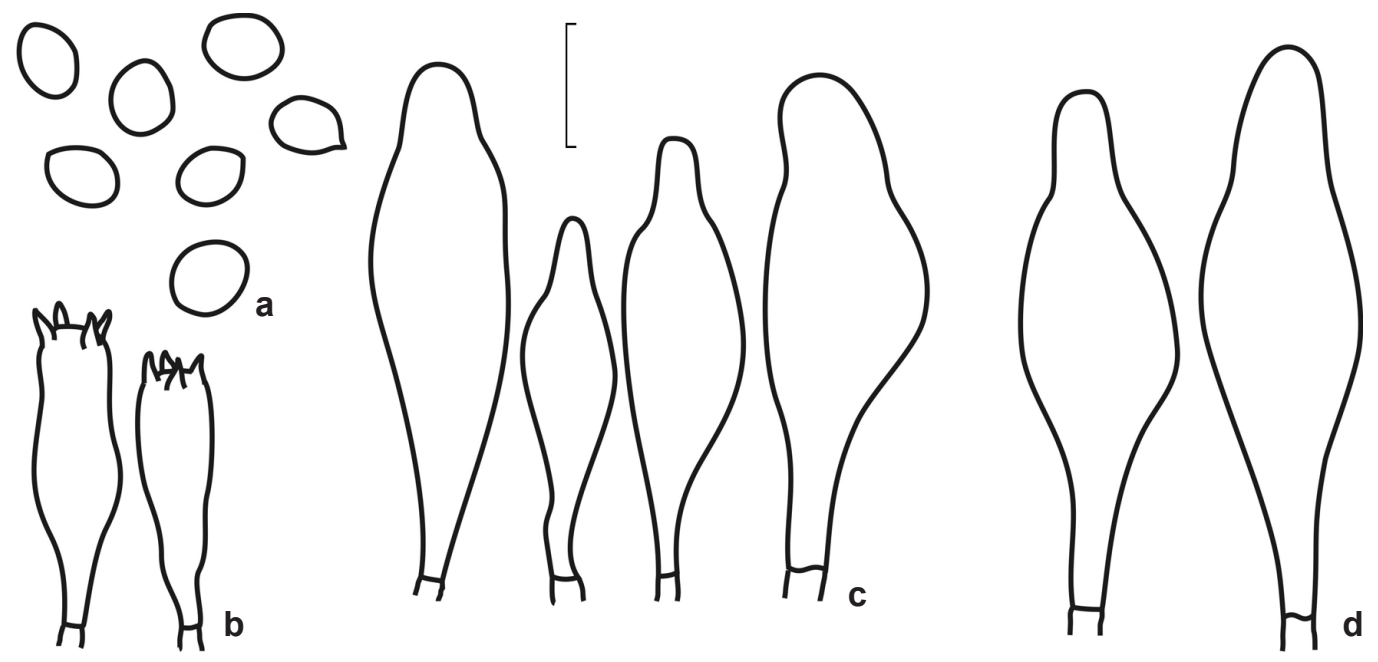

Figura 2 - Volvariella pusilla - a. esporas; b. basidios; c. queilocistidios; d. pleurocistidios. Escala $=10 \mu \mathrm{m}$. Figure 2 - Volvariella pusilla - a. spores; b. basidia; c. pleurocystidia; d. cheilocystidia. Scale $=10 \mu \mathrm{m}$. 
material tipo depositado en el herbario LPS como así también de materiales topotípicos nuevos.

En los materiales analizados observamos pleurocistidios y queilocistidios de menor tamaño que los descritos por Shaffer (1957) y Boekhout (1986, 1990) de materiales de Norteamérica y Europa respectivamente. Estos autores mencionan pleurocistidios abundantes que llegan hasta los $85 \mu \mathrm{m}$ de longitud. Sin embargo, el tamaño y la abundancia de los pleuricistidios coinciden con la descripción dada por Pegler (1983), la cual indica pleurocistidios lageniformes de 36-42 × 11-16 $\mu \mathrm{m}$., no abundantes. Singer \& Digilio (1952) y Spegazzini (1899) no describen detalladamente los pleurocistidios y queilocistidios para los materiales anteriormente descritos de Argentina.

\section{Volvariella subxerophytica Singer, Fieldiana,} Botany 21: 93, 1989.

Figs. 3; 4

Píleo hasta $25 \mathrm{~mm}$ diám., ocasionalmente hasta $30 \mathrm{~mm}$, convexo, con el centro levemente umbonado, frecuentemente con el centro deprimido alrededor del umbo, superficie gris oscuro (6F3) a marrón oscuro (6E6-6E4) hacia el centro, prolongándose en forma de fibrillas hasta un poco más de la mitad del píleo, entremezclándose con el fondo cremeo a castaño claro (4B2-6A2) que predomina en el margen, seco; margen entero, incurvado, estriado a levemente sulcado. Laminillas apretadas a próximas, blancas (1A1), tornándose rosadas (6A3-6A4), margen liso, concoloro con los lados de las laminillas, libres, ventricosas, hasta $5 \mathrm{~mm}$ de ancho, con lemélulas. Estípite central, 25-35 × 3-6 mm, cilíndrico, igual, blanco (1A1),

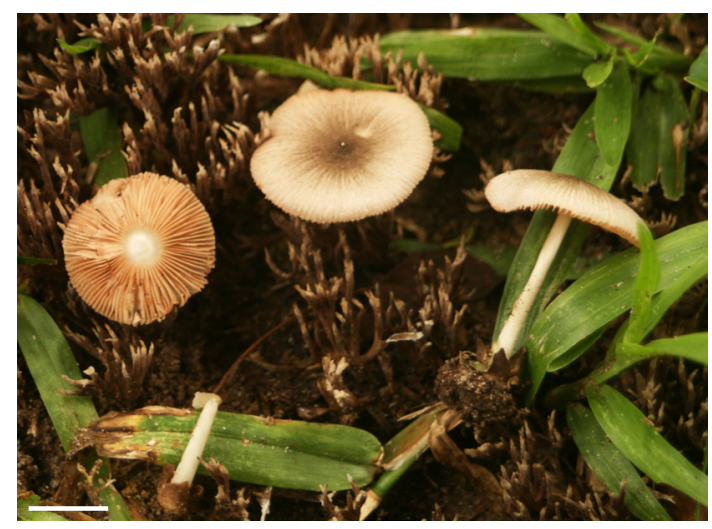

Figura 3 - Aspecto general de Volvariella subxerophytica. Escala $=1 \mathrm{~cm}$.

Figure 3 - General aspect of Volvariella subxerophytica. Scale $=1 \mathrm{~cm}$. glabro. Volva sacciforme, 4-lobada, hasta $12 \times 6$ $\mathrm{mm}$, gris amarronada a marrón oscura (6E7-6E8), glabra a finamente velutina. Tomento basal ausente. Anillo ausente. Esporada rosada pálida.

Esporas (6-)6,5-8,5(-9) × (4,5-)5-6,5 $\mu \mathrm{m}$; $\mathrm{x}=7,3 \times 5,3 ; \mathrm{Q}=1,18-1,57 ; \mathrm{Q}_{\mathrm{x}}=1,35 ; \mathrm{n}=60$; $\mathrm{N}=2$; elipsoidales a anchamente elipsoidales, hialinas a amarillas pálidas, de paredes delgadas, lisas, inamiliodes. Basidios 18-25 × 7-10 $\mu \mathrm{m}$; claviformes, tetraesporados, de paredes delgadas, hialinas. Pleurocistidios ocasionales, muy dispersos y similares a los basidios, 25-35 $\times$ 10-20 $\mu \mathrm{m}$, claviformes a ventricosos, con el ápice anchamente mucronado, sin un contenido distintivo. Queilocistidios no observados. Trama himenoforal convergente, hifas 4-10 $\mu \mathrm{m}$ diám. Pileipellis en un cutis de hifas entremezcladas, 3-10 $\mu \mathrm{m}$ diám. Fíbulas ausentes en todos los tejidos.

Hábitat: Solitario o formando pequeños grupos dispersos, creciendo en el suelo entre la hojarasca.

Distribución: Especie conocida solo para el subtrópico argentino por los materiales descriptos en el presente trabajo.

Material examinado: ARGENTINA. CATAMARCA: Santa María, near Rio Santa María, 27.I.1962, Singer T3752, T3769 (LIL). CORRIENTES: Capital, Facultad de Ciencias Agrarias, II.2011, N. Niveiro 2064 (CTES). SALTA: Guemes. Quebrada de Yaquismé, On Sandy shifting neked soil in subxerophytic forest during rainy period, $900 \mathrm{~m}$ alt., 7.II.1957. R. Singer T2952 (LIL). TUCUMÁN: Pie de Periquillo, $900 \mathrm{~m}$ alt., Ad terram nudam in margine superiore erosionis fluvialis loco subaprico, 30.XII.1951, Singer 1687 (LIL) Tipo. Al lado del camino entre Tapia y Vipos, II.1955. R. Singer \& Digilio (LIL) como Volvariella aff. taylorii.

Observaciones: Volvariella subxerophytica se caracteriza por tener la superficie del píleo grisáceo oscuro a marrón oscuro, que se extiende por fibrillas hasta un poco más de la mitad del píleo, dejando el margen de color castaño claro a cremeo, tiene una volva de color gris oscuro, regularmente lobulada, y el tamaño del basidioma es relativamente pequeño. Es una especie con caracteres intermedios entre $V$. taylorii y $V$. pusilla. Con la primera comparte caracteres como la coloración del centro de la superficie del píleo y el color de la volva, pero se diferencia en que $V$. subxerophytica es de menor tamaño y tiene esporas más grandes (Fig. 5). Con $V$. pusilla comparte el tamaño pequeño de sus basidiomas, el color claro del margen de la superficie del píleo, pero se diferencia en que $V$. pusilla presenta una volva de color claro constante (Shaffer 1957; Boekhout 1986). Volvariella 

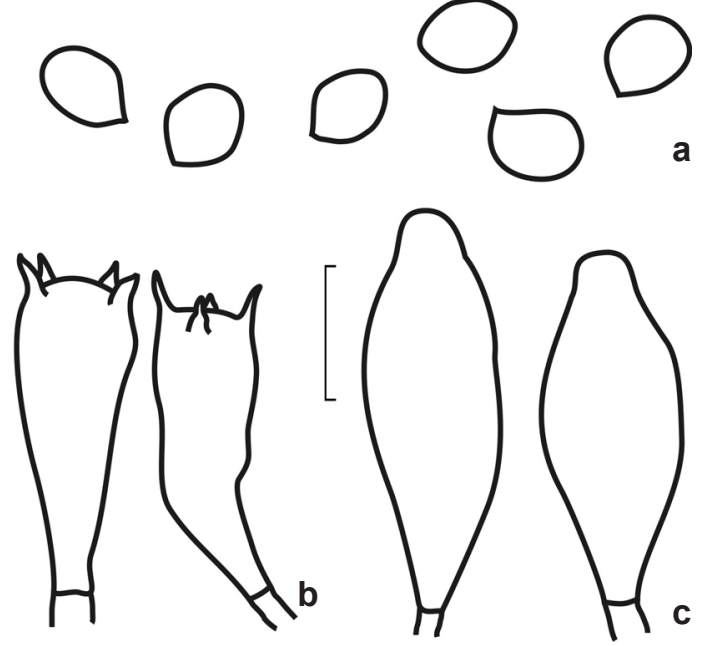

Figura 4 - Volvariella subxerophytica - a. esporas; b. basidios; c. pleurocistidios. Escala $=10 \mu \mathrm{m}$.

Figure 4 - Volvariella subxerophytica - a. spores; b. basidia; c. pleurocystidia. Scale $=10 \mu \mathrm{m}$.

subxerophytica podría confundirse además con $V$. psammophila porque ambas presentan la superficie del píleo con el disco gris oscuro aclarándose hacia el margen dando un aspecto rimoso. Sin embargo, V.psammophila presenta basidiomas más grandes, esporas más pequeñas y numerosos pleurocistidios (Singer 1989).

El material tipo de V. subxerophytica (Singer $\mathrm{T}$ 1687) se encuentra depositado en el herbario de la Fundación Miguel Lillo (LIL) bajo el nombre de Volvariella taylorii. Consiste en un único basidioma completo en relativo buen estado de conservación y un estípite suelto. El píleo se encuentra parcialmente cerrado, lo que nos indica que es un material relativamente joven. La volva es débilmente pilosa, de un color marrón oscuro a casi negro, está presente en uno de los dos estípites que presenta el espécimen tipo y se encuentra parcialmente desprendida. Microscópicamente se observaron esporas anchamente elipsoidales, $7-8,1(-9,1) \times 5-6,2 \mu \mathrm{m}$, basidios claviformes, 4-esporados, de $18-25 \times 7-10 \mu \mathrm{m}$ y pleurocistidios claviformes a ventricosos de 25-35 × 9-20 $\mu \mathrm{m}$, con el ápice ocasionalmente mucronado y pileipellis en un cutis. A diferencia de lo descripto originalmente por Singer en el material tipo (Singer 1989), se han observado pleurocistidios, los cuales podrían ser eventualmente confundidos con basidiolas, pero se diferencian en que son levemente más grandes y ocasionalmente presentan el extremo anchamente mucronado.
El material colectado en la provincia de Corrientes, difiere de las colecciones típicas del noroeste argentino en que presenta basidiomas más grandes, y esporas levemente más pequeñas, caracteres que la asemejan a $V$. taylorii o $V$. psammophila. Sin embargo, por presentar pleurocistidios insconspicuos, no tener la superficie del píleo rimosa hasta el margen y las esporas que en promedio se aproximan más a las del material tipo, consideramos que el material corresponde a V. subxerophytica.

3. Volvariella taylorii (Berk. \& Broome) Singer, Lilloa 22: 401. 1951 [1949]. Agaricus taylorii Berk. \& Broome, Annals and Magazine of Natural History 13: 398. 1854.

Figs. 6; 7

Píleo 20-50 mm de diám., ampliamente cónico, convexo, volviéndose plano-convexo, umbonado, a veces con el centro deprimido alrededor del umbo, llamativamente fibrilloso hasta el margen, gris amarronado a gris claro (6D4-6E5), centro castaño oscuro (6F8), con abundantes fibrillas radiales dejando ver entre ellas el contexto grisáceo (6C4-6D4), seco; margen entero, incurvado, no estriado. Contexto carnoso, delgado, blanco a gris claro (1A1-1B1). Olor y sabor no testeados. Laminillas apretadas a próximas, blancas (1A1), tornándose rosadas (6A4-6B4), margen liso, concoloro con los lados de las laminillas, libres, ventricosas, hasta $5 \mathrm{~mm}$ ancho, con lamélulas. Estípite central, 35-65 × 3-6 mm, cilíndrico, igual, blanco (1A1), glabro. Volva sacciforme, irregularmente lobada, con 1 a 3 lóbulos, hasta $20 \times 12 \mathrm{~mm}$, castaña clara a castaña grisácea (5D4-6D4), glabra o tomentosa. Tomento basal en la base de la volva poco abundante, blanco (1A1). Anillo ausente. Esporada rosada pálida.

Esporas 5-7 $\times(3-) 3,5-4,8 \mu \mathrm{m} ; \mathrm{x}=6,2 \times 4,2$; $\mathrm{Q}=1,2-1,9 ; \mathrm{Q}_{\mathrm{x}}=1,48 ; \mathrm{n}=40 ; \mathrm{N}=2$; oblongas, elipsoidales a ovoides, hialinas a amarillas pálidas, de paredes delgadas, lisas, inamiliodes. Basidios 20-24 × 6-8 $\mu \mathrm{m}$; tetraesporados, claviformes, hialinos, de paredes delgadas. Pleurocistidios $35-$ $49 \times 8-11 \mu \mathrm{m}$, ventricosos a claviformes, con el ápice ocasionalmente mucronado a acuminado, de paredes delgadas, hialinas. Queilocistidios 28-43 $\times 9-13 \mu \mathrm{m}$, claviformes, ocasionalmente con el ápice anchamente mucronado, hialinos, de paredes delgadas, dispersos pero abundantes en el margen de la laminilla. Trama himenoforal convergente, hifas $2-8 \mu \mathrm{m}$ de diám. Pileipellis en un cutis de hifas no gelatinosas, postradas, entremezcladas, 
más o menos paralelas, 3-11 $\mu \mathrm{m}$ de diám., con pigmentos vacuolares grisáceos. Fíbulas ausentes.

Hábitat: Solitario o formando pequeños grupos dispersos, creciendo en el suelo entre la hojarasca.

Distribución: Ampliamente distribuida en regiones templadas de Europa y Norteamérica (Boekhout 1990). Conocida para Europa (Lange 1936; Kuhner \& Romagnesi 1953; Orton 1974), Norteamérica (Shaffer 1957), el Caribe (Pegler 1983). En Sudamérica esta especie fue citada para Argentina por Singer (1953) y Shaffer (1957) en base a especímenes que posteriormente fueron descriptos como V. subxerophytica (Singer 1989). Material examinado: ARGENTINA. MISIONES: Guaraní, Parque Provincial Caá Yarí, sendero del Palmital, en interior de selva (26 $52^{\circ}$ ' 15,5 ' $\mathrm{S}, 5^{\circ}{ }^{\circ} 13^{\prime} 28,9^{\prime}$ 'W, 520 msnm), 20.III.2010, N. Niveiro et al. 1680 (CTES). Reserva de Biosfera Yaboty, Mesa Redonda, en interior de selva (27²'53,9''S, 54'2'17,7''W, $434 \mathrm{msnm})$, 18.IV.2011, N. Niveiro \& O. Popoff 2442 (CTES). General Manuel Belgrano, Reserva Natural Estricta San Antonio (261'33'S, 5347'56'W), 28/04/2015, N. Niveiro et al. 2873 (CTES). JUJUY: Ledesma, Parque Nacional Calilegua, sendero Guaraní (2345'66,1'”S,
64'51'15"W, 627 msnm), 24.III.2011, N. Niveiro et al. 2250 (CTES). SALTA: Santa Victoria Oeste. Reserva Natural El Nogalar de los Toldos, 25.IV.2012, E. Albertó 2704 (CTES). TUCUMÁN?: s/d, Ad humum silvestrem in silva subtropicali, $R$. Singer T2193 (LIL) como Volvariella volvacea vel aff.

Observaciones: Volvariella taylorii se caracteriza por su píleo mediano, fuertemente fibrilloso en sentido radial y por presentar una volva castaña grisácea en la madurez. El material analizado coincide bien con la descripción realizada por Pegler (1983), sin embargo Shaffer (1957) describe, en base a material de Europa, Norteamérica y Argentina, estructuras microscópicas más grandes (esporas, pleurocistidios y queilocistidios). No obstante, esto podría explicarse al considerar que el material que este autor estudió de la Argentina (Singer 1687 LIL) correspondería a lo que actualmente se considera $V$. subxerophytica, el cual tiene estructuras microscópicas más grandes.

Una especie notablemente similar es $V$. leucocalix Sá \& Wartchow recientemente descrita para el noreste brasilero (Sá \& Wartchow 2016).

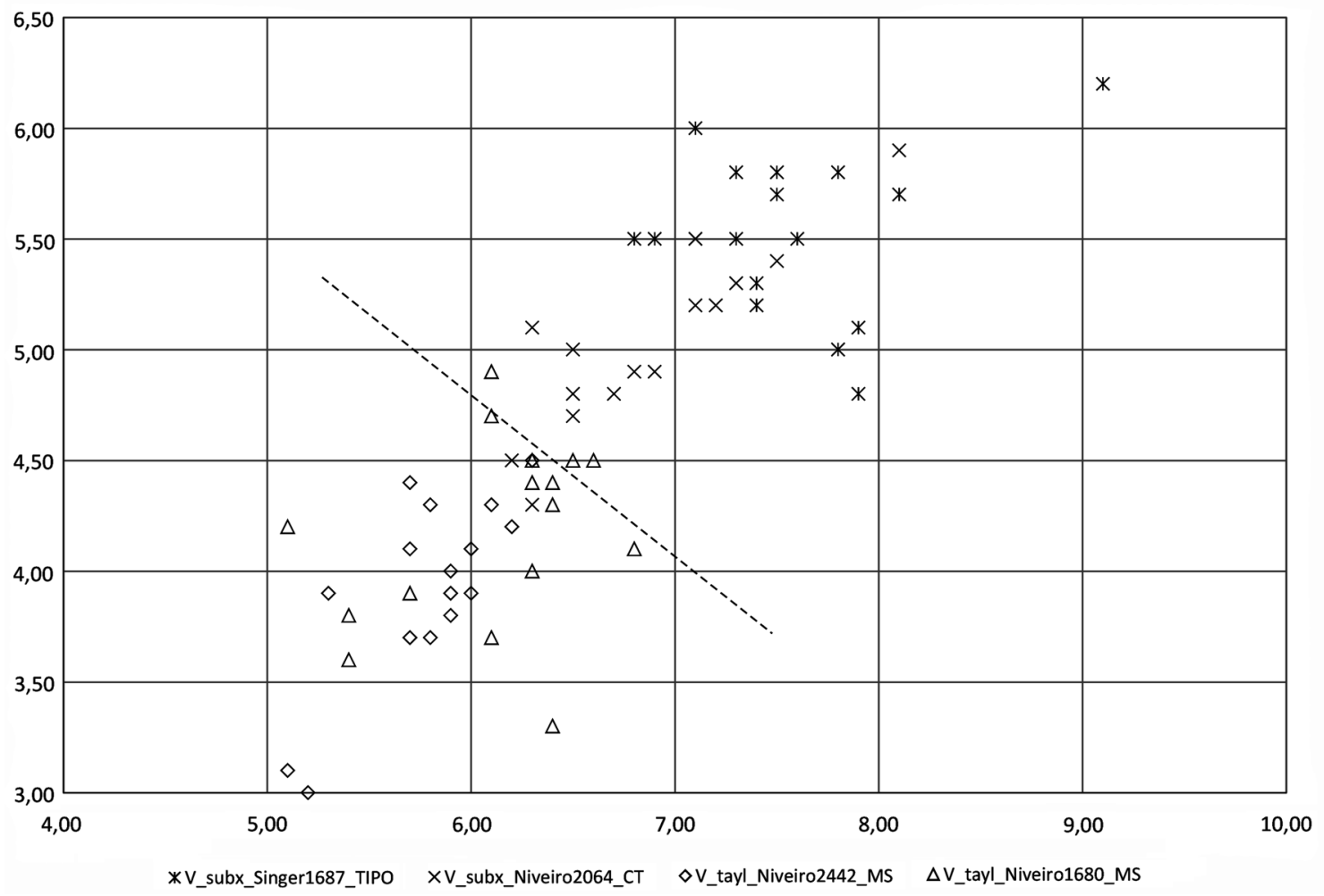

Figura 5 - Diagrama de dispersión del tamaño de esporas de $V$. subxerophytica y $V$. taylorii. Sobre el eje $x$ se indica la longitud de las esporas y sobre el eje $y$ el diámetro.

Figure 5 - Scatter diagram of $V$. subxerophytica and $V$. taylorii spores size. On the $x$-axis the spores' length and on the $y$-axis the spores' diameter are represented. 
Esta especie comparte con V. taylorii el tamaño del basidioma, la superficie radialmente fibrillosa gris oscura y estructuras microscópicas como la forma y tamaño de las esporas y cistidios. Sin embargo, $V$. leucocalix se caracteriza por su volva blanquecina (Sá \& Wartchow 2016), lo que la diferencia de $V$. taylorii que tiene una volva grisácea. Otra especie muy similar es V. lepiotospora Singer, especie poco conocida de Norteamérica, que también presenta basidiomas medianos con fibrillas negras en la superficie del píleo dispuestas radialmente y volva grisácea. Asimismo, Shaffer (1957) diferencia ambas especies por el tamaño de las esporas, siendo las esporas de $V$. taylorii más anchas (4-6 $\mu \mathrm{m}$ diám.) que las de $V$. lepiotospora (3-4 $\mu \mathrm{m}$ diám.). Sin embargo, en los materiales examinados del norte argentino se observaron esporas más delgadas que las descriptas por Shaffer (1957), lo que puede generar una confusión entre ambas especies. No obstante, $V$. lepiotospora se diferencia porque presenta la volva aracnoidea y queilocistidios claviformes (Singer 1955).
Menolli \& Capelari (2008) describen a $V$. heterospora Menolli \& Capellari para Brasil, la cual es similar a $V$. taylorii por su tamaño mediano y la superficie del píleo con fibrillas grises, pero se diferencia notoriamente de esta última por su volva blanca y esporas más grandes y de dos tipos diferentes.

Otra especie similar a $V$. taylorii es $V$. volvacea, pero esta última se diferencia por tener basidiomas más grandes y una volva pilosa más desarrollada (Pegler 1983; Justo \& Castro 2010). Para la Argentina se encontraba registrada $V$. volvacea correspondiente al material Singer T2193 (LIL), sin embargo, al analizar este espécimen, se observó que aunque no se conservan los restos de la volva, presenta los mismos caracteres que los materiales analizados correspondientes a $V$. taylorii.

Boekhout (1986) propone el nombre $V$. pusilla var. taylorii, considerando que las diferencias entre ambas son mínimas. Sin embargo, por el tamaño del basidioma, la coloración de la superficie del píleo
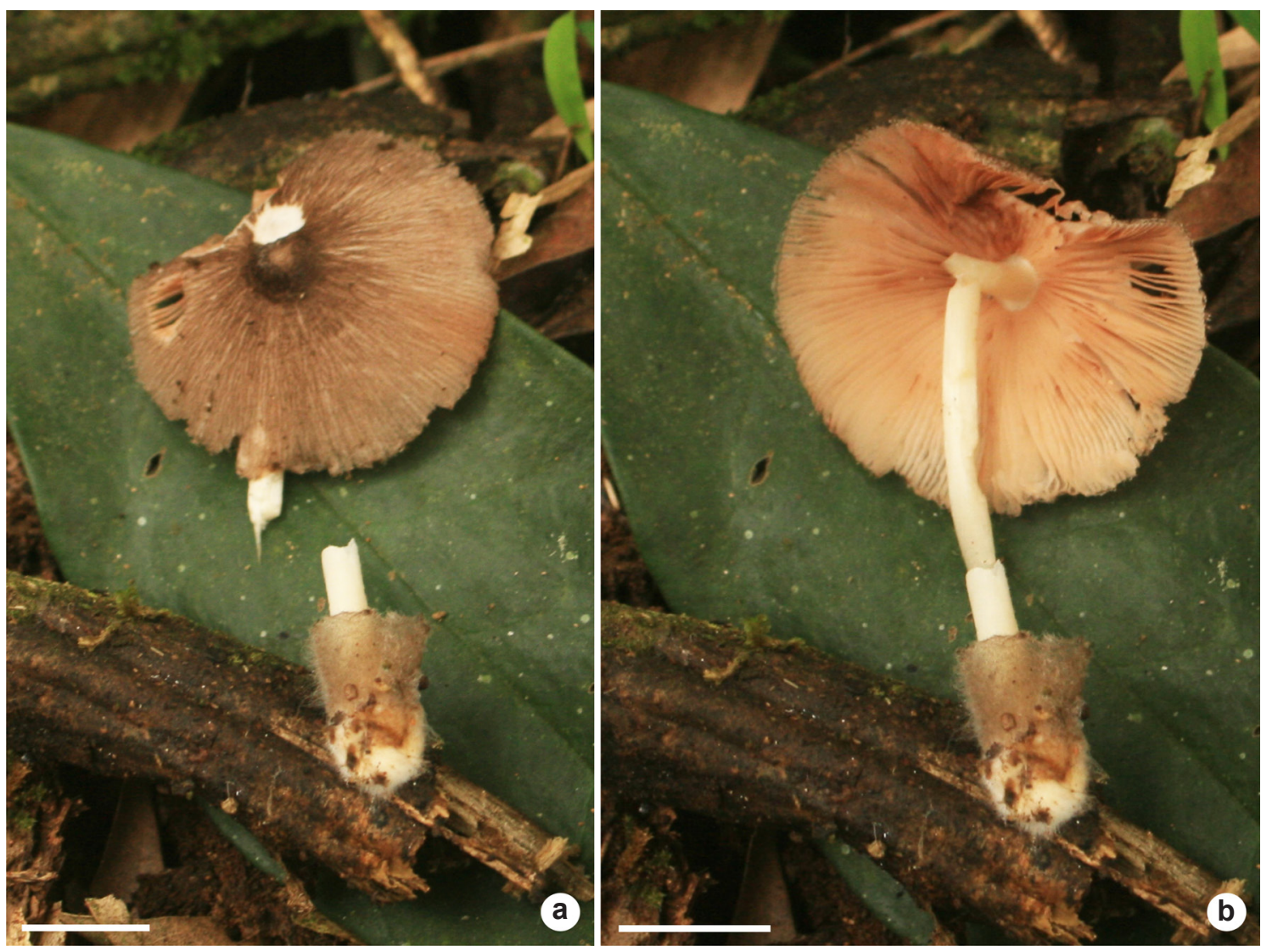

Figura 6 - Volvariella taylorii - a. vista de la superficie del píleo; b. vista de las laminillas. Escala $=1 \mathrm{~cm}$.

Figure 6 - Volvariella taylorii - a. view of pileus surface; b. view of lamellae. Scale $=1 \mathrm{~cm}$. 

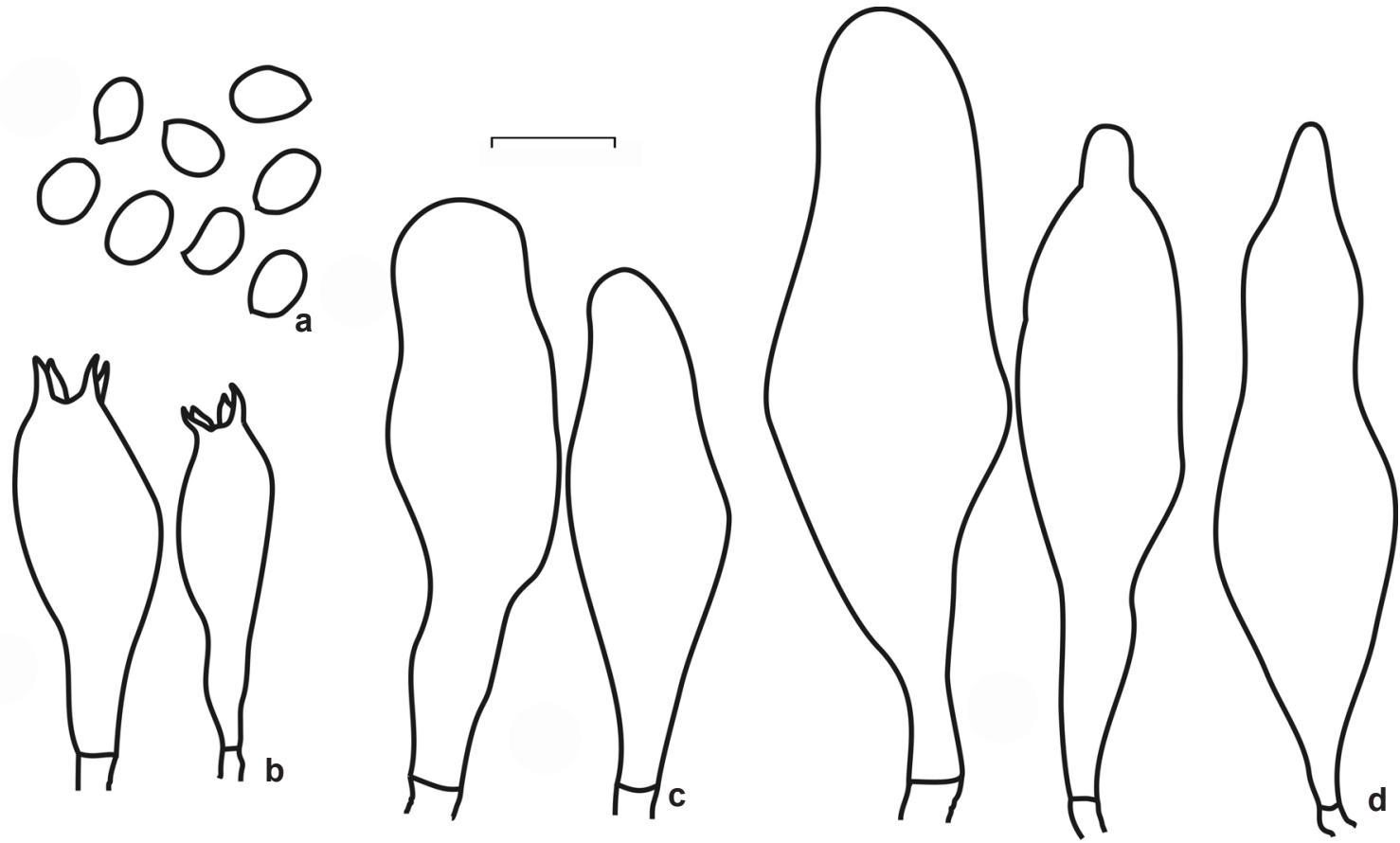

Figura 7 - Volvariella taylorii - a. esporas; b. basidios; c. queilocistidios; d. pleurocistidios. Escala $=10 \mu \mathrm{m}$. Figure 7 -Volvariella taylorii - a. spores; b. basidia; c. cheilocystida; d. pleurocystidia. Scale $=10 \mu \mathrm{m}$.
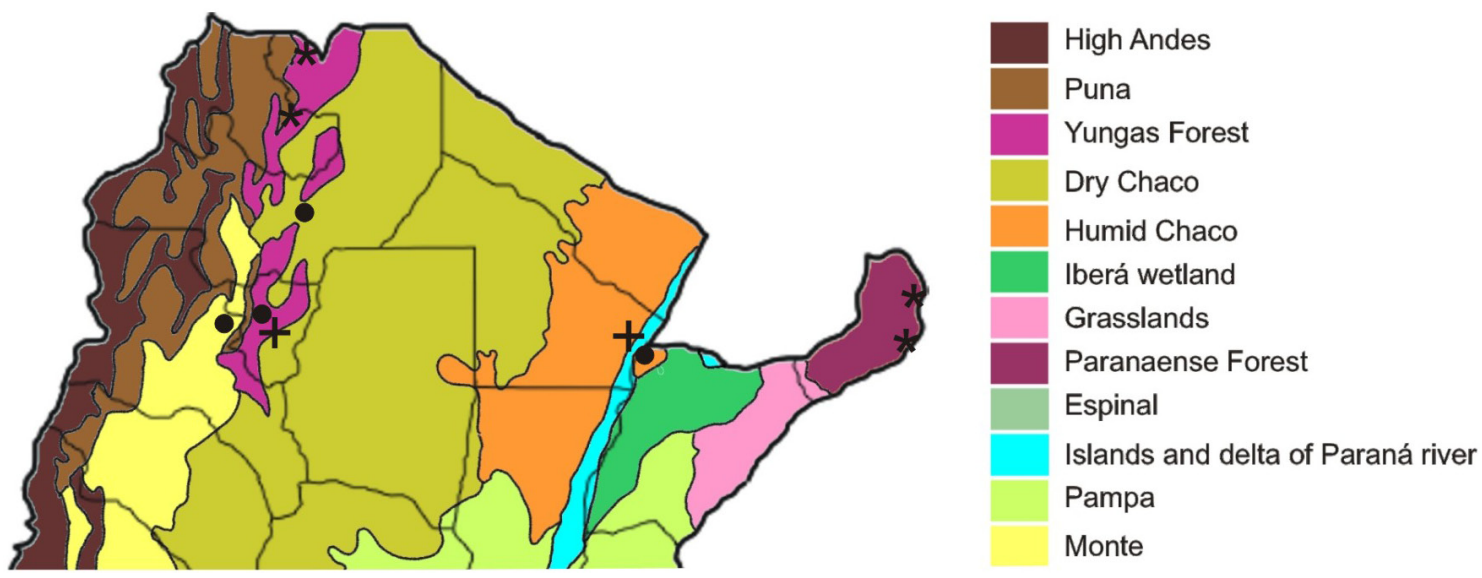

Figura 8 - Mapa de distribución de las especies de Volvariella en el norte de Argentina - (+) V.pusilla; $(\bullet) V$. subxerophytica; $(*) V$. taylorii. Las Ecorregiones corresponden a Burkart et al. (1999).

Figure 8 -Volvariella's species distribution map in northern Argentina - (+) V. pusilla; $(\bullet)$ V. subxerophytica; $(\boldsymbol{*})$ V. taylorii. The ecorregions follow Burkart et al. (1999).

y volva y el tamaño de esporas, las consideramos como especies independientes, muy similares y afines entre sí, pero con caracteres suficientemente diferentes.

Existieron algunas confusiones con respecto a la presencia de esta especie en la Argentina. Singer (1953) registra por primera vez para el país a $V$. taylorii, con material coleccionado de la provincia de Tucumán. Posteriormente, en base al mismo espécimen [Singer 1678 (LIL)] y con la adición de nuevas colecciones de la provincia de Catamarca [Singer T3752, T3769 (LIL)], observa que lo que él originalmente identificó como $V$. taylorii, en realidad representaba una 
especie diferente, $V$. subxerophytica (Singer 1989). Al adicionar nuevas colecciones de estas dos especies y al analizar su distribución se puede observar que $V$. subxerophytica está relacionada a los bosques xerófilos de la región chaqueña, o más específicamente a la distribución de los Bosques Estacionalmente Secos (Prado 2000). Por otro lado, $V$. taylorii, se relaciona con ambientes selváticos, encontrándose en las selvas de Yungas y Paranaense (Fig. 8).

\section{Agradecimientos}

Los autores agradecen al Ministerio de Ecología, Recursos Naturales Renovables y Turismo de la provincia de Misiones y a la Administración de Parques Nacionales por los permisos de colección. Las colecciones obtenidas para el presente trabajo fueron obtenidas gracias a campañas financiadas por el Proyecto Regional Araucaria XXI, Bosque Atlántico-AECID del MERNRyT, por la Secretaria General de Ciencia y Técnica de la Universidad Nacional del Nordeste (SGCyT-UNNE) y por el Consejo Nacional de Investigaciones Científicas y Tecnológicas (CONICET).

\section{Referencias}

Boekhout T (1986) Notulae ad Floram Agaricinam Neerlandicam-XII. Small, saprophytic Volvariella species in the Netherlands. Persoonia 13: 197-211.

Boekhout T (1990) Volvariella. In: Bas C et al. Flora Agaricina Neerlandica 2. A.A. Balkema, Rotterdam. Pp. 56-64.

Burkart R, Bárbaro N, Sánchez R \& Gómez D (1999) Eco-regiones de la Argentina. Administración de Parques Nacionales, Buenos Aires. Pp. 1-43.

Index Fungorum - Authors of fungal names (2016) Disponible en <http://www.indexfungorum.org/ names/AuthorsOfFungalNames.asp $>$. Acceso en 19 Agosto 2016.

Justo A \& Castro M (2010) The genus Volveriella in Spain: $V$. dunensis comb. \& stat. nov. and observations on V. earlei. Mycotaxon 112: 261-270.

Justo A, Vizzini A, Minnis A, Menolli Jr. N, Capelari M, Rodriguez O, Malysheva E, Contu M, Ghignone S \& Hibbett D (2011) Phylogeny of the Pluteaceae (Agaricales, Basidiomycota): taxonomy and character evolution. Fungal Biology 115: 1-20.

Kirk P, Cannon P, Minter D \& Stelpers J (2008) Ainsworth \& Bisby's. Dictionary of the Fungi. $10^{\text {th }}$ ed. CABI, Wallingford. Pp. 1-771.

Kornerup A \& Wanscher J (1978) Methuen Handbook of Colour. Eyre Methuen, London. Pp. 1-252.

Kuhner R \& Romagnesi H (1953) Flore analytique des champignons supérieurs. Masson et. Cie, Paris. Pp. 1-252.
Lange J (1936) Flora agaricina Danica. Recato A/S, Copenhagen. Pp. 1-105.

Largent D (1986) How to identify mushrooms to genus I: macroscopic features. Mad River Press, Eureka. Pp. 1-166.

Largent D \& Baroni T (1988) How to identify mushrooms to genus VI: modern genera. Mad River Press, Eureka. Pp. 1-270.

Menolli Jr. N \& Capelari M (2008) Records and two new species of Volvariella (Pluteaceae, Agaricales) from Brazil. Mycotaxon 106: 385-398.

Moncalvo J, Vilgalys R, Redhead S, Johnson J, James T, Aime M, Hofstetter V, Verduin S, Larsson E, Baroni T, Thorn R, Jacobsson S, Clemencon H \& Miller Jr. O (2002) One hundred and seventeen clades of euagarics. Molecular Phylogenetics and Evolution 23: 357-400.

Niveiro N \& Albertó E (2012) Checklist of the Argentine Agaricales I. Amanitaceae, Pluteaceae and Hygrophoraceae. Mycotaxon 121: 499-500.

Orton P (1974) The European species of Volvariella Spegazini. Bulletin mensuel de la Société linnéenne de Lyon 43: 311-321.

Pegler D (1977) A preliminary Agaric Flora of east Africa. Kew Bulletin Additional Series 6: 1-615.

Pegler D (1983) Agaric flora of the Lesser Antilles. Kew Bulletin, Additional series 9: 1-668.

Prado D (2000) Seasonally dry forest of tropical South America: from forgotten ecosystems to a new phytogeographic unit. Edimburgh Journal of Botany 57: 437-461.

Raithelhuber J (2000) Hongos Agaricales de la Isla Martin García. Metrodiana Sonderheft 5: 1-59.

Raithelhuber J (2004) Nueva Flora Micológica Argentina. Mycosur, Stuttgart. Pp. 1-576.

Sá M \& Wartchow F (2016) Volvariella leucocalix (Pluteaceae), a new species from Brazilian semiarid region. Mycosphere 7: 30-35.

Shaffer R (1957) Volvariella in North America. Mycologia 49: 545-579.

Shaffer R (1962) Synonyms, new combinations, and new species in Volvariella (Agaricales). Mycologia 54: 563-572.

Singer R (1950) Type studies on Basidiomycetes IV. Lilloa 23: 147-246.

Singer R (1953) Quelques Agarics nouveaux de l'Argentine. Revue de mycologie 18: 3-23.

Singer R (1955) New and interesting species of Basidiomycetes. IV. Mycologia 47: 763-777.

Singer R (1986) The Agaricales in modern taxonomy, $4^{\text {th }}$ ed. Koeltz Scientific Books, Koenigstein. Pp. 1-981.

Singer R (1989) New taxa and new combinations of Agaricales (Diagnoses fungorum novorum agaricalium IV). Fieldiana, Botany 21: 1-133.

Singer R \& Digilio A (1952) Pródromo de la Flora Agaricina Argentina. Lilloa 25: 6-461.

Spegazzini C (1899) [1898]. Fungi argentini novi v. critici. Anales Museo Nacional de Historia Natural de Buenos Aires 6: 6-365. 
Spegazzini C (1926) Observaciones y adiciones a la micología argentina. Boletín de la Academia Nacional de Ciencias en Córdoba 28: 267-351.

Thiers B [permanentemente actualizado]. Index Herbariorum: a global directory of public herbaria and associated staff. New York Botanical Garden's
Virtual Herbarium. Disponible en $<\mathrm{http}$ :// sweetgum.nybg.org/ih/>. Acceso en 19 Agosto 2016.

Wright J \& Albertó E (2002) Guía de los hongos de la región Pampeana. I. Hongos con laminillas. L.O.L.A., Buenos Aires. Pp. 1-280. 